\title{
Perception threshold and electrode position for spinal cord stimulation
}

\author{
Jiping He ${ }^{\mathrm{a}, *}$, Giancarlo Barolat ${ }^{\mathrm{a}}$, Jan Holsheimer ${ }^{\mathrm{b}}$ and Johannes J. Struijk ${ }^{\mathrm{b}}$ \\ a Department of Neurosurgery, Thomas Jefferson University, Philadelphia, PA 19107 (USA) and \\ ${ }^{b}$ Department of Electrical Engineering, University of Twente, Twente (The Netherlands)
}

(Received 29 October 1993, revision received 22 February 1994, accepted 23 February 1994)

\begin{abstract}
Summary
The perception threshold for epidural spinal cord stimulation in chronic pain management was analyzed on 3923 testing data obtained from 136 implanted patients. The initial areas of paresthesiae due to stimulation were recorded and reported as the stimulation map according to the location of electrodes. Measurement of dorsal thickness of the cerebrospinal fluid (CSF) layer was obtained from 26 subjects using magnetic resonance imaging (MRI). The results indicate that the perception threshold is a function of the spinal level of the implanted electrodes, of the mediolateral position in the spinal canal and the contact separation of electrode. Differences in perception threshold at various vertebral levels are mainly due to varying depths of the dorsal CSF layer. The medially placed electrodes caudal to the mid-cervical levels have a higher perception threshold than more laterally placed ones. The electrodes at high and mid-cervical levels, however, have a smaller perception threshold if placed medially. The information obtained from this investigation has important implications for the design of a new-generation stimulation system and clinical application to maximize the longevity of the power source.
\end{abstract}

Key words: Perception threshold; Spinal cord stimulation; Electrode combination; Cerebrospinal fluid; Mediolateral position; Pain; Battery life

\section{Introduction}

Application of electrical stimulation to the spinal cord (SCS) for alleviating intractable, chronic pain has received wide acceptance (Long and Erickson 1975; Demirel et al. 1984; Broseta et al. 1986; Meglio et al. 1989; Devulder et al. 1991; Spiegelman and Friedman 1991). The procedure entails implantation of an array of electrodes either surgically or percutaneously into the dorsal epidural space (Racz et al. 1989; Barolat et al. 1991). The electrodes are connected either directly to an implantable stimulation signal generator or radio-frequency coupled to an externally placed stimulation signal generator. The exact neural structures being stimulated, directly and trans-synaptically, are uncertain but include many pathways in both the dorsal and

\footnotetext{
* Corresponding author: Jiping He, Ph.D., Neurosurgery Department, Thomas Jefferson University, 1015 Chestnut Street, Philadelphia, PA 19107, USA. Tel.: (215) 955-4800; FAX: (215) 923-2840.
}

ventral quadrants of the spinal cord (Bantli et al. 1975; Ranck 1975).

Stimulation can reduce the perception of pain by generating paresthesia over the pain area, since the electrical signal will activate large myelinated sensory fibers (Melzack and Wall 1965; Wall and Melzack 1989). Because the activated fibers will be close to the cathodal contact it is important to determine at which spinal level to implant the electrodes, in order to generate the best overlap of paresthesia and pain areas. Reports on the mapping of spinal levels for SCS implant and the paresthesia areas induced by stimulation have been published (North et al. 1991; Barolat et al. 1991, 1993). The corresponding perception threshold and initial distribution of paresthesiae to electrical stimulation at different vertebral levels, however, have not been studied in depth. The perception threshold is the minimum intensity of electrical stimulation at a given vertebral level to induce paresthesiae. A low perception threshold often implies a low requirement for stimulation power, thereby prolonging the battery life. This is especially important for totally implantable 
stimulation systems to reduce the number of surgical procedures for battery replacement. Therefore investigation into which parameters most affect the perception threshold has significant implication for both clinical application and design of new-generation SCS devices.

An important criterion to determine whether an electrode placement is successful is to detect whether the paresthesiae induced by stimulation overlap with the pain area. Even though such overlap does not always relieve pain for patients, little or no overlap means complete failure of the implant. It is a common observation that a laterally placed electrode will generate a unilateral sensation in a radiating band pattern because of the stimulation of the nearest dorsal roots, while a more medially placed electrode will generate paresthesiae bilaterally and in a wider area caudal to the electrode level (Barolat et al. 1993). A thorough investigation of how the mediolateral position and the spinal level of an electrode influence the perception threshold and initial paresthesia will help to determine the success of electrode placement in the initial testing stage.

In this paper we will report our findings from the analysis of 3923 clinical data obtained from testing 136 implanted patients. The results indicate that the perception threshold is a function of the spinal level of electrode placement. The medially placed electrode has a higher perception threshold than does the laterally placed one. The data from MRI of 26 subjects indicate that the perception threshold is largely determined by the dorsal thickness of the CSF layer.

The detailed analysis as discussed in this paper is a step in our effort to establish a knowledge-based expert system. Such a system should contain information to provide a consultation service as advice and guidance to clinicians performing SCS for chronic pain management. Information under consideration includes indications for SCS, pain areas, a possible paresthesiae map, where to place the electrode, what stimulation parameters (amplitude, pulse width and rate) to use, and correlation among the paresthesiae of different body areas, in addition to the parameters discussed in this report. We believe that such an expert system will improve the efficacy of SCS for chronic pain by changing currently a trial-and-error procedure into an experience-guided procedure.

\section{Methods}

\section{Patients}

Patients with chronic pain were implanted with Resume electrodes that contain 4 contacts with a center separation of $10 \mathrm{~mm}$ (Medtronic, Minneapolis, MN). Details of the surgical procedure have been previously reported (Barolat et al. 1991, 1993). Patients age ranges from 19 to 73 years. There were 66 male and 70 female subjects. All the patients were able to perceive tingling paresthesiae and none suffered from major sensory deficit. None of the patients were found to have any abnormality in the epidural space at the level of the implanted electrodes. The patients were affected from chronic pain due to either 'failed back' syndrome or neuropathic pain (reflex sympathetic dystrophy, peripheral nerve injury, etc.) None of the patients included in this study had spinal cord or head injury, plexus avulsion, DREZ, etc., that could affect their paresthesiae response.

\section{Measurement of perception threshold}

To maintain consistency for this study, a standard procedure was followed in all testing and data collection. All data were collected within 10 days from implant. For each patient, all bipolar and unipolar combinations in the implanted electrode were tested. A unipolar combination is a configuration where only a cathode is selected among the 4 contacts while the metal case of the implanted stimulation signal generator is used as the anode. A bipolar combination is that where one contact is selected as the cathode and another as anode. There are 4 unipolar combinations and 12 bipolar combinations for a 4 contacts electrode. A total of 3923 bipolar and unipolar combinations were tested and tabulated for this analysis.

Whereas the amplitude was varied, the other stimulation parameters (rate, pulse width) were maintained constant for standard testing to reduce the number of variables. The stimulation signal was composed of rectangular monophasic pulses at a rate of $50 \mathrm{~Hz}$ and a pulse width of $210 \mathrm{msec}$. The voltage (stimulation amplitude) was gradually increased starting from $0 \mathrm{~V}$ in $0.1-\mathrm{V}$ interval (the minimum amplitude increment for the Medtronic Itrel II pulse generator) or $0.25-\mathrm{V}$ interval (the minimum amplitude increment for the earlier model, the Medtronic Itrel I pulse generator) to detect the perception threshold and maximum stimulation threshold for each combination. The latter is either the maximum voltage the stimulator can generate or the threshold voltage perceived as uncomfortable by the patient, due to excessive sensory stimulation or often caused by involuntary muscle activity. The useful range of stimulation is determined by the difference between these two threshold voltages.

\section{Initial paresthesiae at perception threshold}

The distribution of paresthesiae at the perception threshold stimulation for each electrode combination was recorded on a body map. Twenty-one body areas were used for this analysis (see Fig. 2 for the listing of body areas). For each area ' 1 ' was entered if the patient reported sensation due to stimulation at the perception threshold. Similarly the maximum paresthesiae areas under the maximum stimulation level were also recorded and were reported previously (Barolat et al. 1993).

Following this initial testing phase, the electrical stimulation parameters were adjusted according to the individual requirements in order to obtain the best therapeutic result, which is determined by the best overlap between the paresthesiae and pain areas, a frequency and pulse setting the patient feels most comfortable, and a low voltage to preserve battery life.

\section{Measurement of electrode position}

The vertebral level and mediolateral position of the electrodes in the spinal canal were measured from postoperative $\mathrm{X}$-rays and $\mathrm{CT}$ scans. In the mediolateral direction, a contact was defined as located in the midline of the spinal canal if its center was within $2 \mathrm{~mm}$ either side from the midline. If the center was more than $2 \mathrm{~mm}$ from the midline, the contact was considered as laterally placed.

\section{Measurement of the dorsal thickness of CSF layer}

The distance between the spinal cord and dura mater at the dorsal side of the dural sac was measured from transverse MRI of 
healthy volunteers. Twenty-six nurmal subjects, 19-38 years of age, participated in the study. Series of 6 slices were made from each subject in supine position at 3 vertebral levels: C4-C6, T5-T6 and T11-T12. Each series had the same orientation, perpendicular to the posterior border of the corresponding vertebral bodies. In order to obtain high contrast between spinal tissue, CSF and dura mater, in combination with a high signal-to-noise ratio and short scan time (4.5 min), strongly $\mathrm{T} 2$-weighted Turbo Spin Echo scans were made. The resolution was $0.6 \mathrm{~mm}$. At each vertebral level the thickness of the dorsal CSF-layer was the average from 2 or 3 slices of a subject and was rounded off at $0.5 \mathrm{~mm}$.

The accuracy of the system was tested by measuring the size of a cylindrical vessel placed inside a standard test phantom. The observed error varied between 0 and $2 \%$ in anterior-posterior and transverse directions. A correction for this error has not been made. The stability of the system adjustment was tested once a week, using the manufacturer's test protocol. The variation of the adjustment had a standard deviation of $0.3 \%$. From a few subjects several scans were made in the same or in a different session. No differences between these images could be observed which means that differences were less than 3\% (Holsheimer et al. 1994).

\section{Statistical analysis of data}

In addition to the descriptive statistics that generates mean, median, standard deviation, etc., analysis of variation (ANOVA) was performed to test the statistical significance of any difference between groups. A $P$ value smaller than 0.05 from ANOVA indicates the difference to be statistically significant. The ANOVA was performed on perception thresholds when a difference was observed if data were grouped according to certain parameters, such as mediolateral position of electrodes and contact separation.

The coefficient of correlation was also calculated in this paper to establish relations between 2 different variables, such as the perception threshold and the dorsal thickness of the CSF layer. The larger the coefficient (between 0 and 1), the stronger the relation will be.

\section{Results}

\section{Perception threshold}

The low thoracic spinal levels T9-T11 displayed the largest number of tested combinations, more than 300 at each of those levels. The low cervical to mid-thoracic spinal levels had the least number of tested combinations. The numbers for all spinal levels, including both medially and laterally placed electrodes, are given in column 7 of Table I.

The mean, median and standard deviation of perception threshold at each spinal level is given in columns 2,3 and 4 respectively. The minimum perception threshold reported by patients at all vertebral levels is between 0.1 to $0.25 \mathrm{~V}$ due to the difference in types of pulse generator used. The actual perception threshold could be smaller. Both the low and high ends of the perception threshold distributions found for each spinal level are given in columns 5 and 6 of Table I, respectively.

The mean and median values are presented in Fig. 1. If the data are smoothed, the average perception threshold has a tilted ' $S$ ' shape with the minimum at

\section{TABLE I}

\section{PERCEPTION THRESHOLD AT EACH VERTEBRAL LEVEL}

For each spinal level where electrodes was implanted the statistical analysis of all perception thresholds was performed. The high thoracic levels (T1-T4) have the smallest number of data collected. The median perception thresholds at all spinal levels are smaller than the mean values. They follow the same trend of changes as the mean values do, however. The lowest value is at low cervical to high thoracic spinal levels, the highest value is at middle thoracic spinal levels.

\begin{tabular}{lllllll}
\hline $\begin{array}{l}\text { Vertebral } \\
\text { levels }\end{array}$ & $\begin{array}{l}\text { Mean } \\
(\mathrm{V})\end{array}$ & $\begin{array}{l}\text { Median } \\
(\mathrm{V})\end{array}$ & SD & $\begin{array}{l}\text { Minimum } \\
(\mathrm{V})\end{array}$ & $\begin{array}{l}\text { Maximum } \\
(\mathrm{V})\end{array}$ & $\begin{array}{l}\text { Data } \\
(\mathrm{n})\end{array}$ \\
\hline C1-C2 & 1.13 & 0.75 & 1.01 & 0.25 & 5.00 & 211 \\
C3 & 0.90 & 0.60 & 0.78 & 0.10 & 3.60 & 117 \\
C4 & 0.92 & 0.70 & 0.84 & 0.10 & 4.90 & 161 \\
C5 & 0.67 & 0.50 & 0.44 & 0.20 & 3.00 & 131 \\
C6 & 0.61 & 0.50 & 0.42 & 0.20 & 2.60 & 85 \\
C7 & 0.57 & 0.50 & 0.30 & 0.20 & 1.80 & 74 \\
T1 & 0.59 & 0.50 & 0.23 & 0.10 & 1.30 & 66 \\
T2 & 0.78 & 0.75 & 0.35 & 0.25 & 1.75 & 68 \\
T3-T4 & 1.60 & 1.40 & 1.17 & 0.25 & 4.75 & 59 \\
T5 & 1.61 & 1.00 & 1.33 & 0.30 & 5.00 & 89 \\
T6 & 1.79 & 1.50 & 1.09 & 0.50 & 5.00 & 184 \\
T7 & 1.82 & 1.70 & 0.95 & 0.30 & 5.80 & 230 \\
T8 & 1.61 & 1.50 & 0.90 & 0.20 & 5.00 & 321 \\
T9 & 1.69 & 1.30 & 1.07 & 0.25 & 5.90 & 451 \\
T10 & 1.64 & 1.25 & 1.24 & 0.20 & 7.00 & 647 \\
T11 & 1.29 & 1.00 & 0.85 & 0.20 & 6.20 & 492 \\
T12 & 1.10 & 1.00 & 0.78 & 0.10 & 5.00 & 397 \\
L1 & 1.02 & 0.75 & 0.81 & 0.20 & 5.60 & 140 \\
\hline
\end{tabular}

the low cervical to high thoracic levels (C6-T1) and the maximum at the mid-thoracic levels (T6-T7).

The perception thresholds for medially or laterally

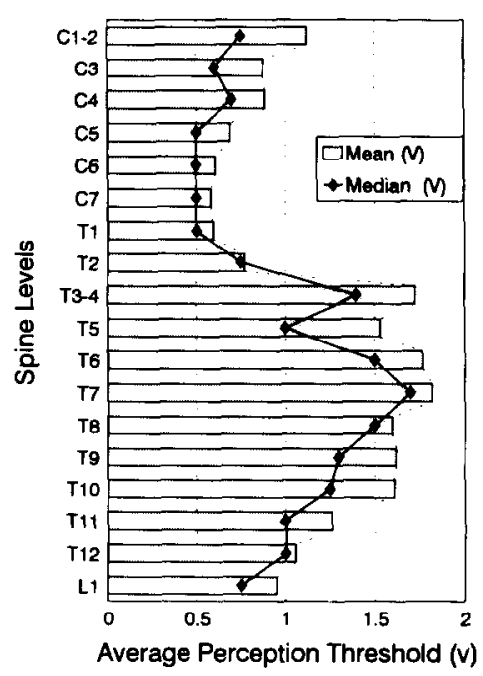

Fig. 1. The average perception thresholds at each vertebral level for epidural dorsal column stimulation. The perception thresholds from all patients are grouped according to the location on vertebral level of electrode contact used as cathode. The perception threshold changes continuously as the vertebral level of the implanted electrode changes from $\mathrm{C} 1$ to L1. The minimum is at C6-T1, the maximum is at levels of T7-T10. 
placed electrodes of all spinal levels were analyzed to examine the effect of the mediolateral position in the spinal canal. The mean value is $1.71 \mathrm{~V}$ for the medially placed electrodes (median: $1.3 \mathrm{~V}$ ) and $1.35 \mathrm{~V}$ for laterally placed ones (median: $1.1 \mathrm{~V}$ ). The $P$ value (from single-factor ANOVA analysis) for our analysis is 0.00035 . The result is given in the column 2 of Table II. The data for this analysis are from more recently implanted patients (after year 1989) to assure a more accurate and consistent measurement of mediolateral positions of electrodes. The total number of combinations included in the analysis is 1279.

Since the uneven distribution of electrodes and the large variation of the perception threshold at different spinal levels, as shown by data in Fig. 1, could distort the analysis result, we analyzed the cffect of the mediolateral position on perception threshold for electrodes at different groups of spinal levels separately. The analysis was performed for electrodes at high to midcervical levels, low cervical to high thoracic levels, mid-thoracic levels and low thoracic levels. The electrodes at T2-T7 were not analyzed because too few laterally placed electrodes were available from our database. The results are given in columns 3-6 of Table II.

When electrodes are implanted caudal to the midcervical levels (C5), the medially placed electrodes have a higher perception threshold than do the more laterally placed ones, a conclusion similar for the general analysis when electrodes at all spinal levels were considered together (Table II). Electrodes at the top of the spinal cord, high to mid-cervical levels (C1-C5), however, have a smaller perception threshold if placed more medially. There were only $7 \%$ of electrodes at C1-C2 that were laterally placed. The perception threshold was smaller for medially placed electrodes at $\mathrm{C} 1-\mathrm{C} 2$ which is consistent with the conclusion for $\mathrm{C} 3$ C5, but $P=0.4>0.05$, therefore we did not include the results in Table II. The $P$ values for all other spinal levels are smaller than 0.05 as shown in the bottom row of Table II.

\section{The paresthesiae map at the perception threshold}

During testing, the stimulation intensity was gradually increased until the patient started to feel paresthesiae. The areas of initial sensation were recorded for each electrode combination. This generated a map linking the location of the electrodes to the paresthesia areas. The maps for medially and laterally placed electrodes were analyzed to investigate possible differences in stimulation patterns. The two maps are given in Fig. 2. For clarity and simplicity of presentation we eliminated some areas and grouped the others to reduce the number of body areas in the figure. The number of electrode combinations available for this analysis was
TABLE II

AVERAGE PERCEPTION THRESHOLDS AT MEDIALLY OR LATERALLY PLACED ELECTRODES

The mediolateral position of electrode in the spinal canal affects the perception threshold. The midline placed electrodes have a higher perception threshold value than do the laterally placed electrodes. High to mid-cervical levels (C1-C5) are an exception. The numbers of data for this analysis are $1279(74 \%$ medial) at all spinal levels, $158(55 \%$ medial) at $\mathrm{C} 3-\mathrm{C} 5,60(63 \%$ medial) at C6-T1, $399(79 \%$ medial) at T8-T10 and $274(72 \%$ medial) at T11-T12.

\begin{tabular}{llllll}
\hline $\begin{array}{l}\text { Electrode } \\
\text { placement }\end{array}$ & \multicolumn{4}{l}{$\begin{array}{l}\text { Average perception thresholds } \\
\text { at various spinal levels }\end{array}$} \\
\cline { 2 - 6 } & $\begin{array}{l}\text { All spinal } \\
\text { levels }\end{array}$ & C3-C5 & C6-T1 & T8-T10 & T11-T12 \\
\hline Midline (V) & 1.71 & 1.03 & 0.62 & 2.34 & 1.69 \\
Lateral (V) & 1.35 & 1.36 & 0.37 & 1.76 & 1.17 \\
$P$ & 0.0004 & 0.0339 & 0.0005 & 0.0050 & 0.0012 \\
\hline
\end{tabular}

1279 because we did not collect the initial paresthesiae for the first 2644 records.

\section{Dorsal thickness of the CSF layer}

The average values and standard deviations for the depth of the dorsal CSF layer were obtained by analyzing MR images at 3 vertebral levels: C4-C6, T5-T6 and T11-T12. The result is presented in Table III.

The curve of the perception thresholds in Fig. 1 suggests that there is a relationship between the thresholds and spinal levels of implanted electrodes. This relationship could be determined by the distance between the nerve fibers in the spinal cord and the electrode contacts. A most significant factor determining this distance could be the thickness of the CSF layer dorsal to the cord.

To test this hypothesis we extracted from Table I the average perception thresholds at the spinal levels for which measurements of dorsal depth of CSF layer were available (see Table III). The perception thresholds and the depths of the dorsal CSF layer were plotted together as shown in Fig. 3. The left vertical axis is for the dorsomedial thickness of the CSF layer in millimeters. The right vertical axis is for the average perception thresholds in volts. The 2 variables have a correlation coefficient of 0.99 , which is significant.

\section{Perception threshold and distance between electrode con-} tacts

One parameter that influences the perception threshold consistently in clinical testing is the distance between the 2 electrical poles of a bipolar combination. To obtain uniform data on the relation between perception threshold and distance between contacts we analyzed data at spinal level T10 where for each distance category there were at least 100 data points (combinations tested). 

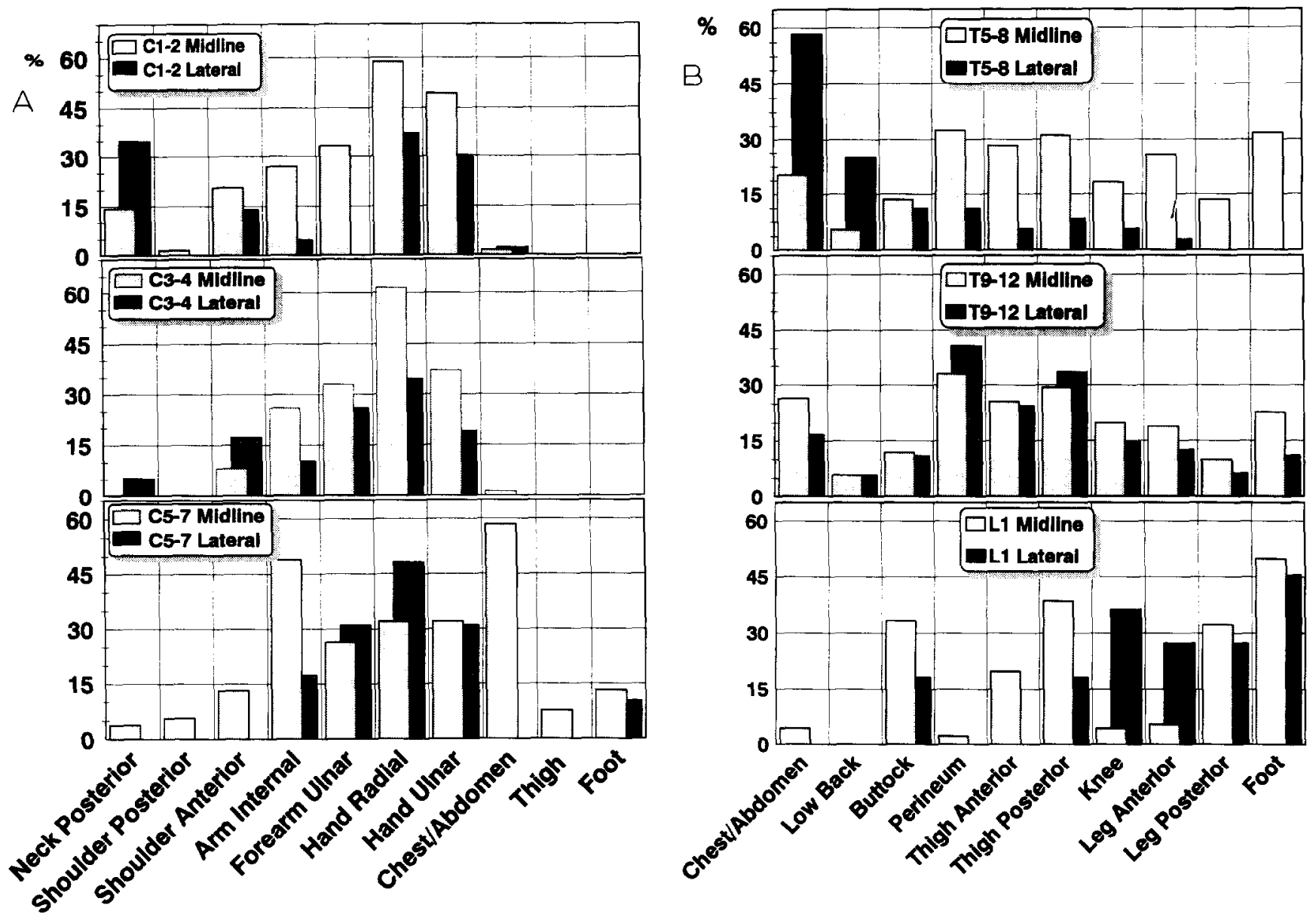

Fig. 2. The stimulation map at the perception threshold level. The percentage of electrodes at each spinal level is given for generating the initial paresthesia at body areas. The dark gray filled bars are for laterally placed electrodes at the given spinal level, the light gray ones are for medially placed electrodes. The vertical axis is the percentage of electrodes eliciting paresthesia in a given body area at the perception threshold. The horizontal axis is the body areas where patients felt paresthesiae at the perception threshold stimulation level.

To further reduce variation, the analysis was performed in 2 steps: intra-subject and then inter-subject. For each patient, the average perception thresholds for different distances between electrical poles (unipolar is considered with an infinite separation) were scaled by the average perception threshold at bipolar separation of $10 \mathrm{~mm}$ ( 2 adjacent contacts). Then the total scaled averagc across all patients was calculated. The number of patients tested for this analysis was 38 .

The result is given in Fig. 4A. The average percep-

\section{TABLE III}

\section{THICKNESS OF DORSAL CSF-LAYER FROM MRI}

The measurement of the thickness of dorsal CSF-layer from MRI is given in this table. For detail of how the values were obtained please see text and (Helsheimer et al., 1993).

\begin{tabular}{lccccccc}
\hline Statistics & C4 & C5 & C6 & T5 & T6 & T11 & T12 \\
\hline Mean (mm) & 2.60 & 2.57 & 2.25 & 5.83 & 5.81 & 3.66 & 3.00 \\
SD & 0.88 & 0.88 & 0.66 & 1.81 & 1.82 & 1.64 & 1.06 \\
Number & 24 & 23 & 24 & 26 & 26 & 25 & 26 \\
\hline
\end{tabular}

tion threshold at each distance between electrode contacts (unipolar, $10 \mathrm{~mm}, 20 \mathrm{~mm}, 30 \mathrm{~mm}$ and beyond) from all tested data is given in Fig. $4 \mathrm{~B}$. The average

Thickness of CSF Layers and Perception Thresholds

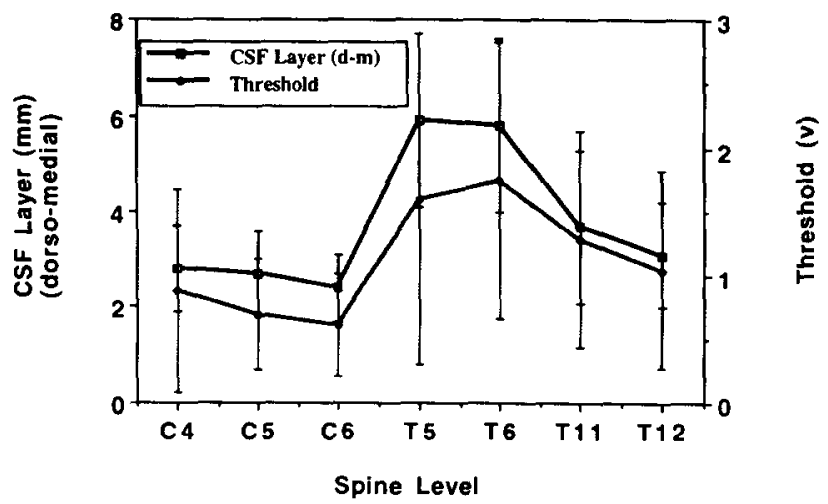

Fig. 3. The correlation of the dorsal thickness of CSF-layers and perception thresholds has a coefficient of 0.99 . The averages and the standard deviations of dorsal depth of CSF-layer and the perception threshold at various vertebral levels were given in the figure. 
A

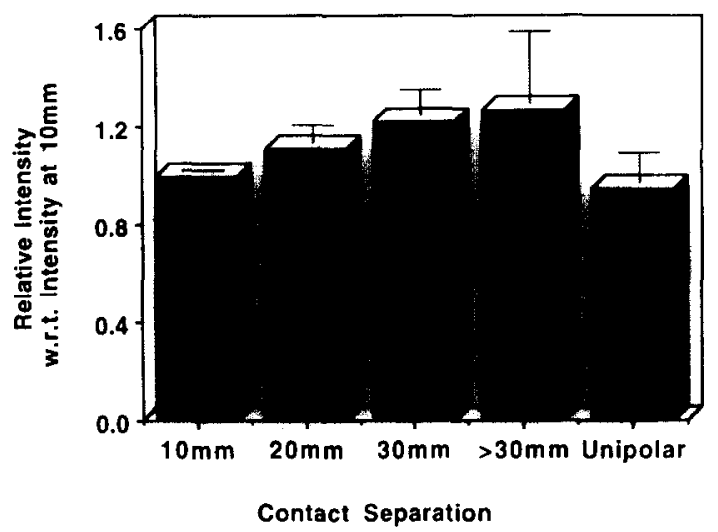

Perception Threshold vs Distance of Contact Separation

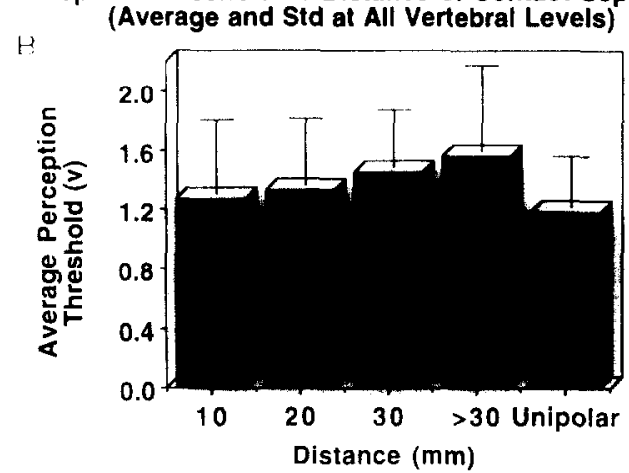

Fig. 4. Statistical relation between contact separation and the perception thresholds is given for both electrodes at Tio (A) and all spinal levels (B). For each patients with T10 stimulation, the perception thresholds were scaled by the value at contact separation of 10 mm to calculate the relative stimulation intensity. Then all patients' data were averaged to present in (A). The data presented in (B) were not scaled as in ( $A$ ). They are the average perception thresholds at each contact separation for electrodes implanted at all spine levels from Cl to L1. The standard deviations are shown by the thin vertical lines.

and standard deviation are given in the figure. Although the differences at various contact separations might not be considered clinically significant, they are statistically significant with $P=0.000136$ (ANOVA) and a confidence level at $90 \%$.

\section{Discussion}

Perception threshold and dorsal thickness of the CSF layer

Data in Fig. 3 clearly indicate that the thickness of the CSF layer at the dorsal side varies along the spinal cord and is an important factor in determining the perception threshold to epidural SCS. It is well known that threshold voltage for the excitation of nerve fibers

\section{TABLE IV}

CONDUCTIVITY OF THE VOLUME CONDUCTOR COMPARTMENTS $\left(\mathrm{S} / \mathrm{m}^{2}\right)$

The CSF has the highest conductivity for SCS. The next is the white matter along its longitudinal direction. The epidural space is mainly the epidural fat. The surrounding layer is everything outside of the vertebral bone.

\begin{tabular}{ll}
\hline Medium & Conductivity \\
\hline Gray matter & 0.230 \\
White matter & \\
$\quad$ Longitudinal & 0.600 \\
$\quad$ Transverse & 0.083 \\
Cerebrospinal fluid & 1.700 \\
Epidural space (fat) & 0.040 \\
Dura mater & 0.030 \\
Vertebral bone & 0.040 \\
Surrounding layer & 0.004 \\
Electrode insulation & 0.001 \\
\hline
\end{tabular}

increases as distance between the electrodes and nerve fiber is increased (Wall and Melzack 1989). The MRI studies show that the thickness of the dorsal CSF layer varies among subjects by more than a factor of 2 at each vertebral level investigated (Holsheimer et al. 1993). Therefore, perception thresholds measured at any level will also vary substantially, as shown in Table I.

In a theoretical study, using a computer model of epidural SCS, we have shown that the variation of perception thresholds among vertebral levels can be explained by the corresponding variation in thickness of the dorsal CSF layer (Struijk et al. 1993a,b). The model comprises the spinal cord (white and gray matter), CSF, epidural space, vertebral bone, electrode insulation, dura mater and a layer representing the surrounding tissues. The conductivity of the compartments is given in Table IV (Struijk et al. 1993a). Volume conductor models representing different levels of spinal cord have different transverse geometry. A transverse section of the model of mid-cervical spinal cord is shown in Fig. 5. The volume conductor was discretized with a rectangular grid with variable grid spacing. The number of grid points was $57 \times 57 \times 57=$ 185193. The model dimensions were $22.4 \times 24.0 \times 60.0$ mm (lateral $\times$ dorso-ventral $\times$ rustro-caudal) (Struijk el al. 1993b).

As shown in Table IV the CSF has by far the highest conductivity. Calculations on the volume conductor model of the spinal cord have shown that $80-$ $90 \%$ of the current between electrode contacts flows through the CSF (when electrode contacts in the epidural space are bordering the dura mater). It is also shown that load resistance increases only slightly with distance between two contacts $(5 \% / \mathrm{cm})$. 
surrounding layer

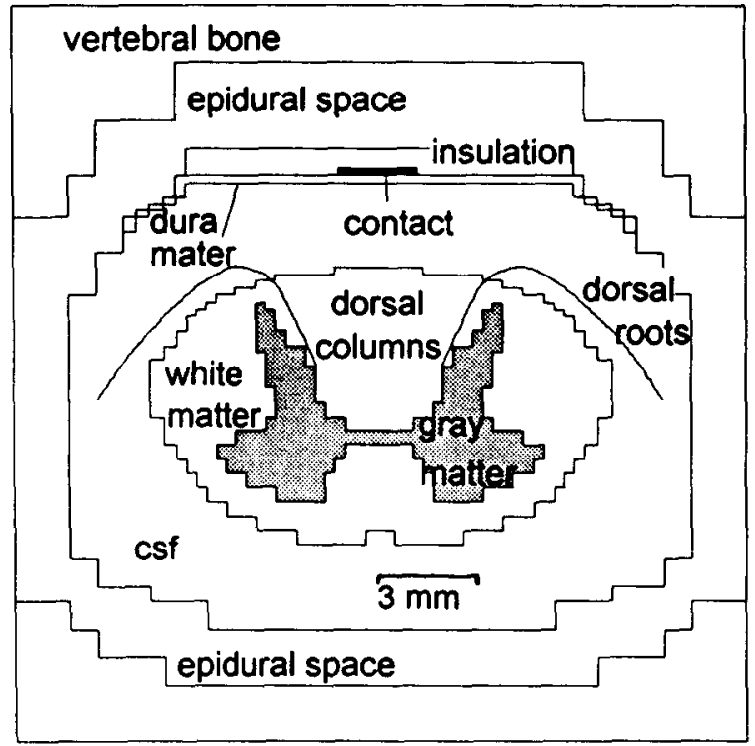

Fig. 5. A transverse section of the volume conductor model at mid-cervical spinal cord. The corresponding conductivity's are in Table IV. The distance from the electrode to the nerve fibers in the spinal cord is mainly determined by the dorsal thickness of CSF-layer.

\section{Perception threshold and contact separation}

The distance between electrode contacts for a bipolar combination influences the threshold as shown by Fig. 4. For all bipolar combinations, the average perception threshold increased with the contact distance.

Although unipolar is the extreme case of bipolar stimulation with the pulse generator as the anode (separation of infinity), the average perception threshold for unipolar is the smallest. This is because the total impedance is much smaller at unipolar stimulation. Two factors contributed to the low impedance in unipolar compared to bipolar stimulation. First, in unipolar stimulation only one lead connection is used instead of two, reducing the impedance by approximately $115 \Omega$. Secondly, the surface area of the anode (metal case of the pulse generator) is much larger than a lead contact. This results in a smaller current density, therefore, less voltage drop near the pulse generator compared to the anode in a bipolar combination. A larger portion of the total stimulation voltage is therefore available under the cathode in unipolar stimulation to stimulate the same nerve fibers.

The modeling study (Struijk et al. 1993a) predicts that perception threshold will increase by approximately $11 \%$ when the contact center separation is increased by $20 \mathrm{~mm}$ from 30 to $50 \mathrm{~mm}$ in computer simulation, which is in accordance with average increase shown in Fig. 4 when the separation is similarly increased from 10 to $30 \mathrm{~mm}$.

In his 1983 report, however, Law found that the perception threshold had a negative correlation with contact separation if cathodes were caudal to anodes while a positive correlation could be found if cathodes were rostral. The difference in conclusion could be explained by differences in approaches for the two studies. Firstly, we did not make a distinction between relative positions of cathodes and anodes as did Law in his study. Secondly, we used the Medtronic Resume electrode while Law implanted 3 percutaneous electrodes in parallel. Therefore our data mostly contained a longitudinal arrangement for the bipolar combinations while Law's data included a large percentage of mediolateral arrangements with a very small longitudinal separation between a cathode and anode pair. Finally, the contact separation in a Resume electrode is typically in $10-\mathrm{mm}$ increments whereas the increment between contacts in 3 parallelly arranged electrodes can be as small as 3-5 mm (Law 1983).

\section{Initial paresthesiae and laterality of electrode}

Stimulation at the upper (C1-C2) and middle (C3-C4) cervical levels generates initial sensation mostly in upper extremities and neck and shoulder areas (Fig. 2A, the top and middle graphs). Stimulation at the lower cervical (C6-C7) and upper thoracic (T1-T4, not shown due to small number of cases available) levels generates initial scnsations mostly in upper extremitics and chest-abdominal area (Fig. 2A, bottom graph). Stimulation at the middle (T5-T8) and lower (T9-T12) thoracic levels generates the initial paresthesia mostly in the lower trunk and lower extremities (Fig. 2B, top and middle graphs). The bottom graph in Fig. 2B gives the map of the initial paresthesia when stimulating at upper lumbar levels (L1).

There were differences between the stimulation maps by medially (light gray bars in Fig. 2) and laterally (dark gray bars) placed electrodes. While the laterally placed electrodes often stimulate areas described as a radiating band pattern, presumably the stimulation of dorsal root fibers in the dorsal root entry zone, the medially placed electrodes stimulate a wider area caudal to the spinal level of the electrodes, even at the perception threshold. A higher stimulation intensity, however, is required to elicit the initial paresthesia for medially placed electrode (Table II).

Another observation is that body areas notoriously difficult to stimulate are seldom activated at the perception threshold level. This is shown by the low occurrence of those areas in the map: posterior shoulder, low back and perineum, and posterior side of lower extremities. Unfortunately these are also the pain areas patients complain most. A larger usage range will be desirable under this situation because the stimulation intensity can be increased gradually to spread the paresthesiae to cover pain areas. Furthermore it would be very helpful to have information available on corre- 
lation of paresthesia at different parts of the body under a given stimulation. With such information one should be able to predict whether a stimulation could reach the desired areas for reducing pain by degree of correlation of initial paresthesia areas and pain areas.

\section{Perception threshold and laterality of electrode}

While the medially placed electrode can stimulate a larger body area than laterally placed electrode does, in general, however, it requires a higher stimulation threshold, as indicated by data in Table II. The difference between the medially and laterally placed electrodes is both statistically $(P<0.05)$ and clinically ( $>$ $0.1 \mathrm{~V}$ ) significant (see below for the exception at high cervical levels). A theoretical study based on a computer model predicts that in both cases dorsal root fibers have lower threshold stimuli than dorsal column fibers (Struijk et al. 1993a). When the electrode was in a medial position the difference between threshold stimuli of dorsal root fibers and dorsal column fibers was less than with a lateral electrode position. Therefore, a larger paresthesia coverage will be possible with a medial electrode position without causing discomfort, if taking into account the limited usage range of the stimulation.

The above discussion will not apply to the electrodes at high to mid-cervical levels, however. Patients received stimulation at high cervical levels showed high sensitivity for medially placed electrodes. The combined benefit of a lower perception threshold and a larger paresthesiae area suggests that a medial position of electrodes at high to mid-cervical levels should be adopted when possible. The high mobility of spine at this level has a tendency to produce changing paresthesiae when patients move or rotate their heads. This further suggests the importance of a medial placement of electrode.

The perception threshold and the initial sensation areas are valuable information in helping to determine at the testing stage whether the placement of an electrode is optimal. If the perception threshold is too high, it suggests that either the connection or electrode placement is problematic. If the initial sensation area is a unilateral radiating band pattern, it indicates an excessively lateral placement of the electrode. It is often desirable to place the electrode as close to the midline as possible (Law 1983; Barolat et al. 1993) even if the topography of pain is initially on one side because this provides a higher uscful range for stimulation (Law 1983). This higher usage range provides extra flexibility in adjusting stimulation parameters to achieve better coverage of topography of pain and comfortable sensation.

In summary, the results from analysis of our data indicate that perception threshold is a function of the spinal level and the mediolateral position of the im- planted electrode, and of the distance between electrode contacts. Variation in the perception threshold along different levels of spinal cord is mainly influenced by the dorsal depth of CSF layer. A midline placement of electrodes and a larger separation between electrode contacts both contributed to a higher perception threshold, except at high to mid-cervical levels. The initial paresthesiae at the perception threshold could reach a wide body area both caudal and rostral to the spinal level of stimulation if electrodes are placed on the midline of the spinal canal. The information presented here should help clinicians to develop a strategy of electrode placement that maximizes the battery life of a totally implantable stimulation system.

\section{Acknowledgements}

We would like to acknowledge the contribution of Beth Ketcik, R.N., Fulvio Massoro, M.D., and Zhuang $\mathrm{Yu}, \mathrm{M} . \mathrm{S}$., in data collection and database management, and Jacques den Boer, Ph.D., and Fred Rozeboom, M.D., in developing the MRI-TSE protocols. This investigation was supported in part by Medtronic (Minneapolis, MN), and in part by the Medisearch Foundation (Enschede, The Netherlands).

\section{References}

Bantli, H., Bloedei J.R., Long, D.M. and Thienprasit, P., Distribution of activity in spinal pathways evoked by experimental dorsal column stimulation, J. Neurosurg., 42 (1975) 290- 295.

Barolat, G., Zeme, S. and Ketcik, B., Multifactorial analysis of epidural spinal cord stimulation. Stereotact. Funct. Neurosurg., 56 (1991) 77-103.

Barolat, G., Massaro, F., He, J., Zeme, S. and Ketcik, B., Mapping of sensory responses to epidural stimulation of the intraspinal neural structures in man. J. Neurosurg., 78 (1993) 233-239.

Broseta, J., Barbera, J., De Vera, J.A., Salorio, J.L., Garcia-March, G., Gonzales-Darder, J., Rovaina, F. and Joanes, V., Spinal cord stimulation in peripheral arterial disease: a cooperative study, J. Neurosurg., 64 (1986) 71-80.

Demirel, T., Braun, W. and Reimers, C.D., Results of spinal cord stimulation in patients suffering from chronic pain after a two year observation period, Neurochirurgia, 27 (1984) 47-50.

Devulder, J., DeColvenaer, L., Rolly, G., Caemaert, J., Calliauw, L. and Martens, F., Spinal cord stimulation in chronic pain therapy, Clin. J. Pain, 6 (1991) 51-56.

Holsheimer, J., den Boer, J.A., Struijk, J.J. and Rozeboom, A.R., Assessment of the normal position of the spinal cord in the spinal canal by MR imaging. Am. J. Neuroradiol, in press.

Law, J.D., Spinal stimulation: statistical superiority of monophasic stimulation of narrowly separated, longitudinal bipoles having rostral cathodes, Appl. Neurophysiol., 46 (1983) 129-137.

Long, D.M. and Erickson, D., Stimulation of the posterior columns of the spinal cord for relief of intractable pain, Surg. Neurol, 4 (1975) 134-142.

Meglio, M., Cioni, B. and Rossi, G.F., Spinal Cord Stimulation in management of chronic pain, J. Neurosurg., 70 (1989) 519-524. 
Melzack, R. and Wall, P.D., Pain mechanisms: a new theory, Science, 150 (1965) 971-979.

North, R.B., Ewend. M.G., Lawton, M.T. and Piantadosi, S., Spinal cord stimulation for chronic, intractable pain: superiority of 'multichannel' devices, Pain, 44 (1991a) 119-130.

North, R.B., Ewend, M.G., Lawton, M.T., Kidd, D.H. and Piantadosi, S., Failed back surgery syndrome: five-year follow-up after spinal cord stimulator implantation, Neurosurgery, 28 (1991b) 692-699.

Racz, G.B., McCarron, R.F. and Talboys, P., Percutanepus dorsal column stimulator for chronic pain control, Spine, 14 (1989) 1-4.

Ranck Jr., J.B., Which elements are excited in electrical stimulation of mammalian nervous system, Brain Res., 98 (1975) 417-440.
Spiegelman, R. and Friedman, R., Spinal Cord Stimulation: a contemporary series, Neurosurgery, 28 (1991) 65-71.

Struijk, J.J., Holsheimer, J., Barolat, G., He, J. and Boom, H.B.K., Paresthesia thresholds in spinal cord stimulation: a comparison of theoretical results with clinical data, IEEE Trans. Rehab. Eng., $(1993 \mathrm{a})$ in press.

Struijk, J.J., Holsheimer, J. and Boom, H.B.K., Excitation of dorsal root fibers in spinal cord stimulation: a theoretical study. IEEE Trans. Biomed. Eng., (1993b) in press.

Wall, P.D. and Melzack, R., Text Book of Pain. Churchill Livingstone, Edinburgh, 1989. 